\title{
Target Mass Corrections in Diffractive Scattering
}

\author{
J. Blümlein ${ }^{1}$, B. Geyer ${ }^{2}$ and D. Robaschik ${ }^{3 *}$ \\ 1- Deutsches Elektronen-Synchrotron, DESY \\ Platanenallee 6, D-15738 Zeuthen, Germany \\ 2- Center for Theoretical Studies and Institute of Theoretical Physics Leipzig University, \\ Augustusplatz 10, D-04109 Leipzig, Germany \\ 3- Brandenburgische Technische Universität Cottbus, Fakultät 1, \\ PF 101344, D-03013 Cottbus, Germany
}

\begin{abstract}
We describe the twist- 2 contributions to inclusive unpolarized and polarized deepinelastic diffractive scattering in an operator approach. The representation refers to the observed large rapidity gap but does not require reference to a pomeron picture. We discuss both the case of vanishing target mass $M$ and momentum transfer $t$ as well as the effects at finite $t$ and $M$, which lead to modifications at large $\beta$ and low values of $Q^{2}$.
\end{abstract}

\section{Introduction}

Deep-inelastic diffractive scattering is one of the important scattering processes in highenergy ep scattering at HERA. In the small- $x$ domain $\sim 1 / 8$ of the events are due to this process. It is characterized by inclusive hadron-production with a large rapidity gap between the outgoing proton and all the remainder hadrons. In Refs. [1] two of the present authors developed a description for this process based on the Compton operator and using techniques known in non-forward scattering, cf. [2], for the case that the momentum transfer $t$ between the incoming and the outgoing proton and target masses can be neglected. In the region of smaller values of $Q^{2}$ and large values of $\beta=x / x_{P}$ one expects both finite $t$ and $M^{2}$ effects which were worked out in [3] based on related investigations for the non-forward case [4], see also [5]. In this paper we summarize the main findings of these analyzes, cf. also [6].

\section{General Structure}

The hadronic tensor of the process is determined by three vectors $p_{1}, p_{2}, q$, the incoming and outgoing proton momentum and the momentum transfer in the unpolarized case supplemented by the spin vector of the initial proton $S$ in the polarized case. The following invariants are formed

$Q^{2}=-q^{2}, \quad W:=\left(p_{1}+q\right)^{2}, \quad x:=\frac{Q^{2}}{Q^{+} W^{2}-M^{2}}, \quad t:=\left(p_{2}-p_{1}\right)^{2}, \quad x_{P}:=-\frac{2 \eta}{2-\eta} \geq x$.

The hadronic tensors in case of the unpolarized and polarized cases are of the following form, [1]

$$
W_{\mu \nu}^{u n p}=\left(-g_{\mu \nu}+\frac{q_{\mu} q_{\nu}}{q^{2}}\right) W_{1}+\hat{p}_{1 \mu} \hat{p}_{1 \nu} \frac{W_{3}}{M^{2}}+\hat{p}_{2 \mu} \hat{p}_{2 \nu} \frac{W_{4}}{M^{2}}+\left(\hat{p}_{1 \mu} \hat{p}_{2 \nu}+\hat{p}_{2 \mu} \hat{p}_{1 \nu}\right) \frac{W_{5}}{M^{2}}
$$

*This paper was supported in part by SFB-TR-9: Computergestütze Theoretische Teilchenphysik. 


$$
\begin{array}{rrr}
W_{\mu \nu}^{p o l} & =i\left[\hat{p}_{1 \mu} \hat{p}_{2 \nu}-\hat{p}_{1 \nu} \hat{p}_{2 \mu}\right] \varepsilon_{p_{1}, p_{2}, q, S} \frac{\hat{W}_{1}}{M^{6}}+ & i\left[\hat{p}_{1 \mu} \varepsilon_{\nu S p_{1} q}-\hat{p}_{1 \nu} \varepsilon_{\mu S p_{1} q}\right] \frac{\hat{W}_{2}}{M^{4}} \\
+ & i\left[\hat{p}_{2 \mu} \varepsilon_{\nu S p_{1} q}-\hat{p}_{2 \nu} \varepsilon_{\mu S p_{1} q}\right] \frac{\hat{W}_{3}}{M^{4}}+ & i\left[\hat{p}_{1 \mu} \varepsilon_{\nu S p_{2} q}-\hat{p}_{1 \nu} \varepsilon_{\mu S p_{2} q}\right] \frac{\hat{W}_{4}}{M^{4}} \\
+ & i\left[\hat{p}_{2 \mu} \varepsilon_{\nu S p_{2} q}-\hat{p}_{2 \nu} \varepsilon_{\mu S p_{2} q}\right] \frac{\hat{W}_{5}}{M^{4}}+i\left[\hat{p}_{1 \mu} \hat{\varepsilon}_{\nu p_{1} p_{2} S}-\hat{p}_{1 \nu} \hat{\varepsilon}_{\left.\mu p_{1} p_{2} S\right] \frac{\hat{W}_{6}}{M^{4}}}\right. \\
+ & i\left[\hat{p}_{2 \mu} \hat{\varepsilon}_{\nu p_{1} p_{2} S}-\hat{p}_{2 \nu} \hat{\varepsilon}_{\left.\mu p_{1} p_{2} S\right] \frac{\hat{W}_{7}}{M^{4}}+}+i \varepsilon_{\mu \nu q S} \frac{\hat{W}_{8}}{M^{2}}\right.
\end{array}
$$

with $\hat{p}_{2 \mu}, \hat{\varepsilon}_{\nu p_{1} p_{2} S}$, etc. the corresponding gauge-invariant completions. In general there are 4 unpolarized and 8 polarized structure functions in case of pure photon exchange.

The twist-2 contributions can be described applying the factorization theorem. Moreover, A. Mueller's generalized optical theorem allows to turn the isolated final state proton into an initial state anti-proton, being separated from the proton by $t$. In this way the diffractive state is formed, from which the hadronic tensor is obtained taking the forward expectation value of the Compton-tensor. Evaluating the process further using the above kinematic variables we are led to a description of the diffractive scattering cross section which does not require any reference to a pomeron picture, but is solely based on the presence of a large rapidity gap.

\section{The Case $t=M^{2}=O$}

In this approximation the number of structure functions reduces to two unpolarized and two polarized ones, because of the collinearity of $p_{1}$ and $p_{2}$, [1]. Due to this the diffractive state simplifies and leads to a Lorentz structure with lower complexity. For pure photon exchange only the structure functions $F_{1,2}$ resp. $g_{1,2}$ contribute, which in the twist-2 approximation obey a modified Callan-Gross relation

$$
F_{2}\left(\beta, \eta, Q^{2}\right)=2 x F_{1}\left(\beta, \eta, Q^{2}\right)
$$

and the Wandzura-Wilczek, respectively. As shown in [1], the evolution equations, changing $x \rightarrow \beta$ are the same as for inclusive deep-inelastic scattering. To derive the diffractive evolution equations one considers the evolution equations for non-forward scattering Ref. [2b]

$$
\mu^{2} \frac{d}{d \mu^{2}} O^{A}\left(\kappa_{+} \tilde{x}, \kappa_{-} \tilde{x} ; \mu^{2}\right)=\int D \kappa^{\prime} \gamma^{A B}\left(\kappa_{+}, \kappa_{-}, \kappa_{+}^{\prime}, \kappa_{-}^{\prime} ; \mu^{2}\right) O^{B}\left(\kappa_{+}^{\prime} \tilde{x}, \kappa_{-}^{\prime} \tilde{x} ; \mu^{2}\right)
$$

which turn into

$$
\mu^{2} \frac{d}{d \mu^{2}} f^{A}\left(\vartheta, \eta ; \mu^{2}\right)=\int_{\vartheta}^{-\operatorname{sign}(\vartheta) / \eta} \frac{d \vartheta^{\prime}}{\vartheta^{\prime}} P^{A B}\left(\frac{\vartheta}{\vartheta^{\prime}}, \mu^{2}\right) f_{B}\left(\vartheta^{\prime}, \eta ; \mu^{2}\right)
$$

in the case $t, M^{2} \rightarrow 0$. The value of $\vartheta$ is determined by the absorptive condition as $\vartheta=2 \beta$. 


\section{Target Mass Corrections}

At low values of $Q^{2}$ and large values of $\beta$ both target mass and finite $t$-effects become important. As shown in Ref. [3], following [4], these effects have to be dealt with together. The method is a generalization of the treatment of target mass effects in [7] to the nonforward case. The now more complicated diffractive states $\left\langle p_{1},-p_{2}, t\right|$ imply that the preparton densities emerging in this case depend on two light-cone variables $z_{ \pm}$, non of which can be integrated out. For further treatment we define the variables

$$
\vartheta=z_{-}+\frac{z_{+}}{\eta}, \quad \zeta=\frac{z_{-}}{\vartheta} .
$$

The presence of the variable $\zeta$ implies that the full Lorentz structure outlined above contributes, assuming azimuthal angular integrals are not carried out. Four unpolarized and eight polarized structure functions contribute. The partonic description being possible in the case $t, M^{2} \rightarrow 0$ at the level of observables does not hold anymore in this case, since $p_{1}$ and $p_{2}$ are no longer collinear. Instead, one has to perform definite integrals (the $\zeta$-integrals in [3]) over pre-partonic two-particle correlation functions, which cannot be determined by experiment directly. The absorptive condition in the present case is given by

$$
\vartheta=-\frac{2 \beta}{\kappa} \frac{1}{1+\sqrt{1+4 \beta^{2} \mathcal{P}^{2}(\eta, \zeta, t) / Q^{2}}} .
$$

Here $|\mathcal{P}(\eta, \zeta)|$ takes the role of the nucleon mass in the case of forward scattering. It holds $\mathcal{P}^{2}=t(1-\zeta / \eta)+\left(4 M^{2}-t\right) \zeta^{2} \geq 0$. As an example, we present the $M^{2}$ and $t$ corrections for the un-integrated unpolarized structure functions $F_{1,2}^{a}[3]$ :

$$
\begin{aligned}
& F_{1}^{a}(\vartheta, \zeta) \equiv \Phi_{a}^{(0)}(\vartheta, \zeta)+\frac{\kappa \mathcal{P}^{2}}{\left[(q \mathcal{P})^{2}-q^{2} \mathcal{P}^{2}\right]^{1 / 2}} \Phi_{a}^{(1)}(\vartheta, \zeta)+\frac{\kappa^{2}\left[\mathcal{P}^{2}\right]^{2}}{(q \mathcal{P})^{2}-q^{2} \mathcal{P}^{2}} \Phi_{a}^{(2)}(\vartheta, \zeta) \\
& F_{2}^{a}(\vartheta, \zeta) \equiv \Phi_{a}^{(0)}(\vartheta, \zeta)+\frac{3 \kappa \mathcal{P}^{2}}{\left[(q \mathcal{P})^{2}-q^{2} \mathcal{P}^{2}\right]^{1 / 2}} \Phi_{a}^{(1)}(\vartheta, \zeta)+\frac{3 \kappa^{2}\left[\mathcal{P}^{2}\right]^{2}}{(q \mathcal{P})^{2}-q^{2} \mathcal{P}^{2}} \Phi_{a}^{(2)}(\vartheta, \zeta)
\end{aligned}
$$

Here the $\zeta$-dependent distribution functions $\Phi_{a}^{(k)}(\vartheta, \zeta)$ are iterated integrals of the correlation function $\Phi_{a}^{(0)}(\vartheta, \zeta)=f_{a}(\vartheta, \zeta)$, cf. [3]. a denotes the respective kinematic invariant, implying kinematic dependences in general.

Although no partonic description is obtained one still may study, whether twist-2 relations between structure functions exist. In case of the Callan-Gross relation this is not expected, since it is absent also for forward scattering [7]. However, the Wandzura-Wilczek relation between the twist-2 contributions of the polarized structure functions $g_{1}$ and $g_{2}$ holds also in the diffractive case for finite values $M^{2}, t$, as in many other cases [8-10]. Here the $\zeta$-integral can be carried out. ${ }^{\mathrm{a}}$ This is not the case for other structure functions. Below this integral, however, all the different structure functions can be represented by a single $\zeta$-dependent two-particle distribution function in the unpolarized and polarized case, respectively. The different $\zeta$-dependence of the respective pre-factors and the fact that the $\zeta$-integral is definite prevents to access the corresponding pre-parton distribution functions.

\footnotetext{
${ }^{a}$ It would be interesting to see, whether the generalization of integral relations derived for the forward polarized case for the twist -2 and twist -3 contributions $[8,9]$ can be generalized to diffractive scattering for electro-weak boson exchange.
} 
At the twist-2 level diffractive parton distributions exist whenever the $M^{2}, t \rightarrow 0$ approximation holds. For large values of $\beta$ and small values of $Q^{2}$ this is not the case. This is also the kinematic region in which one expects higher twist operators to contribute in the light cone expansion. ${ }^{\mathrm{b}}$

The twist-2 scaling violations of the diffractive structure functions in case of $M^{2}, t$ being finite are different from those in the limit $M^{2}, t \rightarrow 0$. Unlike the case there, the non-forward evolution equations do not simplify in the same way and the $\zeta$-dependence will remain here too.

\section{Conclusions}

Deep-inelastic diffractive scattering can be described taking the expectation value of the Compton Operator between the diffractive states $\left\langle p_{1}, p_{2} ; t\right|$ obtained by applying A. Mueller's generalized optical theorem. In the limit $M^{2}, t \rightarrow 0$, two polarized and two unpolarized structure functions contribute to the scattering cross section at twist $\tau=2$. They are related by a modified Callan-Gross relation (in lowest order), resp. the Wandzura-Wilzcek relation in all orders. Target mass corrections accounting for all $M^{2}, t$-effects are required in the region of large values of $\beta$ and low values of $Q^{2}$. The set of genuine diffractive structure functions becomes larger due to these effects: four unpolarized structure functions and eight polarized structure functions (with one relation) contribute. These structure functions can be decomposed into generally different diffractive parton densities due to the $\zeta$-integral. In the case of $M^{2}, t \rightarrow 0$ the scaling violations of the twist $\tau=2$ contribution to the diffractive structure functions are described by the evolution equations for forward scattering replacing $x \rightarrow \beta$. The present approach results into a thorough description demanding a rapidity gap without any need to invoke a "pomeron".

\section{References}

[1] J. Blümlein and D. Robaschik, Phys. Lett. B 517 (2001) 222. Phys. Rev. D 65 (2002) 096002.

[2] D. Müller, D. Robaschik, B. Geyer, F. M. Dittes and J. Horejsi, Fortsch. Phys. 42 (1994) 101. J. Blümlein, B. Geyer and D. Robaschik, Nucl. Phys. B 560 (1999) 283 ;

A. V. Belitsky and A. V. Radyushkin, Phys. Rept. 418 (2005) 1.

[3] J. Blümlein, B. Geyer and D. Robaschik, Nucl. Phys. B 755 (2006) 112.

[4] B. Geyer, D. Robaschik and J. Eilers, Nucl. Phys. B 704 (2005) 279.

[5] A. V. Belitsky and D. Müller, Phys. Lett. B 507 (2001) 173.

[6] Slides: http://indico. cern. ch/contributionDisplay $\cdot$ py? contribId=133\&sessionId=4\&conf Id=9499

[7] H. Georgi and H. D. Politzer, Phys. Rev. D 14 (1976) 1829.

[8] J. Blümlein and N. Kochelev, Phys. Lett. B 381 (1996) 296; Nucl. Phys. B 498 (1997) 285.

[9] J. Blümlein and A. Tkabladze, Nucl. Phys. B 553 (1999) 427.

[10] J. D. Jackson, G. G. Ross and R. G. Roberts, Phys. Lett. B 226 (1989) 159;

A. Piccione and G. Ridolfi, Nucl. Phys. B 513 (1998) 301;

J. Blümlein and D. Robaschik, Nucl. Phys. B 581 (2000) 449;

B. Geyer and M. Lazar, Phys. Rev. D 63 (2001) 094003;

J. Blümlein, V. Ravindran and W. L. van Neerven, Phys. Rev. D 68 (2003) 114004;

B. Geyer and D. Robaschik, Phys. Rev. D 71 (2005) 054018.

[11] K. Golec-Biernat and A. Luszczak, arXiv:0704.1608 [hep-ph].

${ }^{\mathrm{b}}$ For a phenomenological higher-twist approach see [11]. 